\title{
Use of Bersons-Kulsh Form Factor of Short-Pulse Approximation in Rydberg-State Physics
}

\author{
R. PARzyński* AND M. Sobczak \\ Faculty of Physics, A. Mickiewicz University \\ Umultowska 85, 61-614 Poznań, Poland
}

(Received September 10, 2002)

\begin{abstract}
The experiments on high Rydberg states interacting with short electromagnetic pulses were hitherto mainly explained by using numerical integration of the time-dependent Schrödinger equation in a restricted state basis. In this study we apply a different approach based on the Bersons-Kulsh analytical form factor of the short-pulse approximation. This analytical approach is shown to well reproduce the recent experimental results and those of numerical integration of the time-dependent Schrödinger equation both in the case of terahertz half-cycle pulses and optical many-cycle pulses. This fact enables a recommendation of the analytical Bersons-Kulsh form factor as an alternative and efficient method of quantum calculations of electromagnetically induced Rydberg state redistribution.
\end{abstract}

PACS numbers: $32.80 . \mathrm{Rm}, 03.65 . \mathrm{Ge}$

\section{Introduction}

Half-cycle pulses (HCP's) of unipolar electromagnetic radiation from the terahertz frequency regime are now in increasing use in the experimental Rydberg-state physics (e.g. [1]). To be specific, in one of the latest experiment in this field [2], a pair of time-delayed twin HCP's was used to first create in rubidium a Rydberg wave packet around the initial $n=40$ state and then to study the dynamics of the formed wave packet by applying the time-domain Ramsey interference technique. It has been recently shown [3] that the Ramsey

*corresponding author; e-mail: parzynsk@main.amu.edu.pl 
interferograms (state-population versus delay time) from the above experiment could be theoretically well described with no need of numerical solution of the time-dependent Schrödinger equation (TDSE). Instead, an analytical approach combining the short-pulse approximation for the transition amplitude between Rydberg states [4], the Bersons-Kulsh closed-form analytic expression for this amplitude in the parabolic state basis [5] and the unitary transformation of the parabolic Bersons-Kulsh amplitudes to the spherical basis [6] was shown to be successful to the same extent as the numerical solution of TDSE in the above case.

The aim of the present paper is twofold. First of all, we wish to show how well the above analytical approach works when applied to other recent HCP+Rydberg atom experiments, the Ahn et al. experiment on caesium [7] and that by Tielking and Jones on sodium [8]. In the experiment [7], an initial Rydberg wave packet was reduced by a HCP to a practically single state, marked by having its phase flipped with respect to the phases of other states in the original packet. The experiment in [8] involved a measurement of redistribution of the initial single-state population over neighbouring states by a HCP versus electric field strength of the HCP. In both cases, our results obtained from the analytical approach will be shown to agree, at least qualitatively, with the experimental results and those of numerical integration of TDSE. Moreover, we will also show that our previous numerical-experiment results on Rydberg state redistribution by a many-cycle pulse from the optical frequency domain [9] are perfectly reproduced by the present analytic approach.

\section{Theoretical background}

The short-pulse approximation [4] requires for the pulse duration, $t_{\mathrm{p}}$, to be much shorter than the Kepler period, $t_{\mathrm{K}}=2 \pi n^{3}$ a.u., of the Rydberg electron in its initial state so the spectral band width of the pulse covers a number of Rydberg states in the vicinity of the initial one. This pulse transfers a momentum to the electron, which in a.u. amounts to $\boldsymbol{Q}=\left(\varepsilon_{0} / \gamma\right) S$, where both $\varepsilon_{0}$ (electric field amplitude) and $\gamma$ (the rate of changes of the electric field) are given in a.u. of electric field and frequency, respectively, and $S$ is the pulse area obtained by time integration. The above $\boldsymbol{Q}$ determines the Rydberg-to-Rydberg transition amplitude from $|i\rangle$ to $|f\rangle$ through the form factor

$$
T_{f i}=\langle f|\exp (\mathrm{i} \boldsymbol{Q} \boldsymbol{\rho})| i\rangle,
$$

where $\rho$ stands for the electron radius in a.u.

Though the form factor like the above one was extensively studied in the past in the context of collision theory (e.g. [10,11]) only Bersons and Kulsh succeeded recently [5] in finding, for a $z$-directed electric field, a convenient analytic expression for it in parabolic basis: $|i\rangle=\left|n n_{1} n_{2} m\right\rangle$ and $|f\rangle=\left|n^{\prime} n_{1}^{\prime} n_{2}^{\prime} m^{\prime}=m\right\rangle$, where $n_{1}$ and $n_{2}$ are the parabolic quantum numbers interrelated through $n_{1}+n_{2}+|m|+1$ 
$=n$, where $0 \leq n_{1} \leq n-|m|-1[6]$. For the $m=0$ states, the case of interest throughout the present paper, the Bersons-Kulsh expression reads

$$
\begin{aligned}
& T_{n^{\prime} n_{1}^{\prime} n_{2}^{\prime} 0, n n_{1} n_{2} 0} \\
& \quad=\mathrm{i} 2 y z \frac{\left(n-n^{\prime}-\mathrm{i} y\right)^{n_{1}}\left(n^{\prime}-n-\mathrm{i} y\right)^{n_{1}^{\prime}}\left(n-n^{\prime}+\mathrm{i} y\right)^{n_{2}}\left(n^{\prime}-n+\mathrm{i} y\right)^{n_{2}^{\prime}}}{\left(n+n^{\prime}-\mathrm{i} y\right)^{n_{1}+n_{1}^{\prime}+1}\left(n+n^{\prime}+\mathrm{i} y\right)^{n_{2}+n_{2}^{\prime}+1}} \\
& \quad \times\left[\frac{2 n n^{\prime}\left(n_{1}-n_{2}+\mathrm{i} y\right)+\left(n_{2}^{\prime}-n_{1}^{\prime}\right)\left(n^{2}+n^{\prime 2}+y^{2}\right)}{\left(n+n^{\prime}\right)^{2}+y^{2}}\right. \\
& \quad \times F\left(-n_{1},-n_{1}^{\prime}, 1 ; z\right) F\left(-n_{2},-n_{2}^{\prime}, 1 ; z\right) \\
& \quad+n_{1}^{\prime} F\left(-n_{1},-n_{1}^{\prime}+1,1 ; z\right) F\left(-n_{2},-n_{2}^{\prime}, 1 ; z\right) \\
& \left.\quad-n_{2}^{\prime} F\left(-n_{1},-n_{1}^{\prime}, 1 ; z\right) F\left(-n_{2},-n_{2}^{\prime}+1,1 ; z\right)\right]
\end{aligned}
$$

where

$$
y=Q n n^{\prime}, \quad z=-\frac{4 n n^{\prime}}{\left(n-n^{\prime}\right)^{2}+y^{2}},
$$

$Q=|\boldsymbol{Q}|$ and $F(a, b, c ; z)$ is a hypergeometric function.

From the above Bersons-Kulsh expression one can readily find the form factor between spherical states, $|i\rangle=|n l m=0\rangle$ and $|f\rangle=\left|n^{\prime} l^{\prime} m^{\prime}=0\right\rangle$, because the spherical and parabolic bases are interrelated by a unitary transformation [6]. The result of this transformation is

$$
\begin{aligned}
& T_{n^{\prime} l^{\prime} 0, n l 0}=\sum_{n_{1}^{\prime}=0}^{n^{\prime}-1} \sum_{n_{1}=0}^{n-1}(-1)^{n+n^{\prime}+l+l^{\prime}+n_{1}+n_{1}^{\prime}} \\
& \quad \times C_{\left(n^{\prime}-1\right) / 2}^{l^{\prime} 0}\left(2 n_{1}^{\prime}-n^{\prime}+1\right) / 2\left(n^{\prime}-1\right) / 2\left(-2 n_{1}^{\prime}+n^{\prime}-1\right) / 2 \\
& \quad \times C_{(n-1) / 2}^{l} 0 \\
& \quad \times T_{n^{\prime} n_{1}^{\prime} n_{2}^{\prime}=n^{\prime}-n_{1}^{\prime}-10, n n_{1} n_{2}=n-n_{1}-10,}
\end{aligned}
$$

where $C_{a \alpha b \beta}^{c \gamma}$ means the Clebsch-Gordan coefficients.

\section{Applications}

We apply Eqs. (2)-(4) to first describe the results of the experiment by Ahn et al. [7] performed on the caesium wave packet. The initial packet was created in this experiment by a sculpted optical pulse to include the $|n l=1 \mathrm{~m}=0\rangle$ states with $24 \leq n \leq 29$, i.e, $\sum_{n=24}^{29} a_{n}|n 10\rangle$, where the normalized amplitudes $\left(\sum_{n}\left|a_{n}\right|^{2}=1\right)$ were: $a_{24}=0.24, a_{25}=0.48, a_{26}=0.57, a_{27}=0.48$, $a_{28}=0.33$ and $a_{29}=0.24$ thus forming nearly Gaussian population distribution around $n=26$. This wave packet was then allowed to freely evolve in the time $t$ 

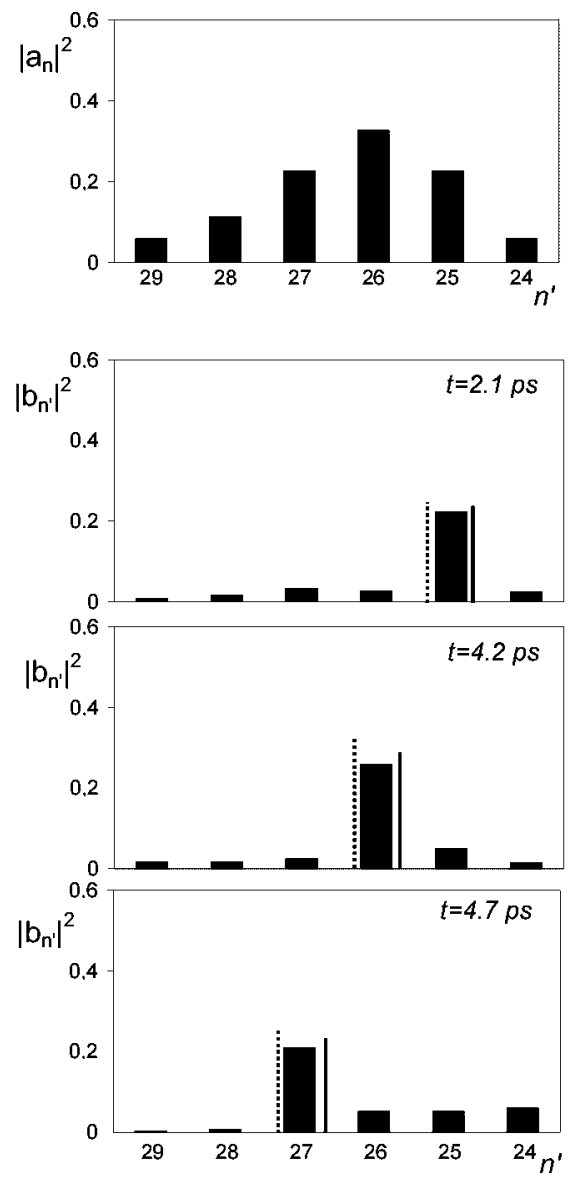

Fig. 1. State population in the initial caesium $p$-type wave packet, $\left|a_{n}\right|^{2}$, and the $p$-state redistribution by terahertz HCP, $\left|b_{n^{\prime}}\right|^{2}$, for different times $t$ of the initial wave-packet free evolution. The results obtained on the basis of Eqs. (4) and (2) (bold bars) are compared with the theoretical data of Ahn et al. obtained by partial-wave expansion for form factor of short-pulse approximation (solid line) and numerical integration of TDSE (dashed line). The comparison is shown for the phase-flipped state in the initial wave packet which survives HCP.

of the order of several picoseconds, much shorter than the lifetimes of the wave packet components but long enough for the components to acquire significant phases, $\omega_{n} t$, resulting in $\sum_{n=24}^{29} a_{n}|n 10\rangle \exp \left(-i \omega_{n} t\right)$. The last wave packet, with different phases of its components, was finally kicked by a terahertz HCP of 0.44 ps duration $\left(t_{\mathrm{p}} / t_{\mathrm{K}} \approx 0.25\right)$ which transferred $Q=0.0043$ a.u. of momentum to the atom. After the HCP, the populations in the $p$ states forming the initial wave packet were measured by the state selective field ionization versus time $t$ of free evolution. Within the short-pulse approximation, justified by the present relation 

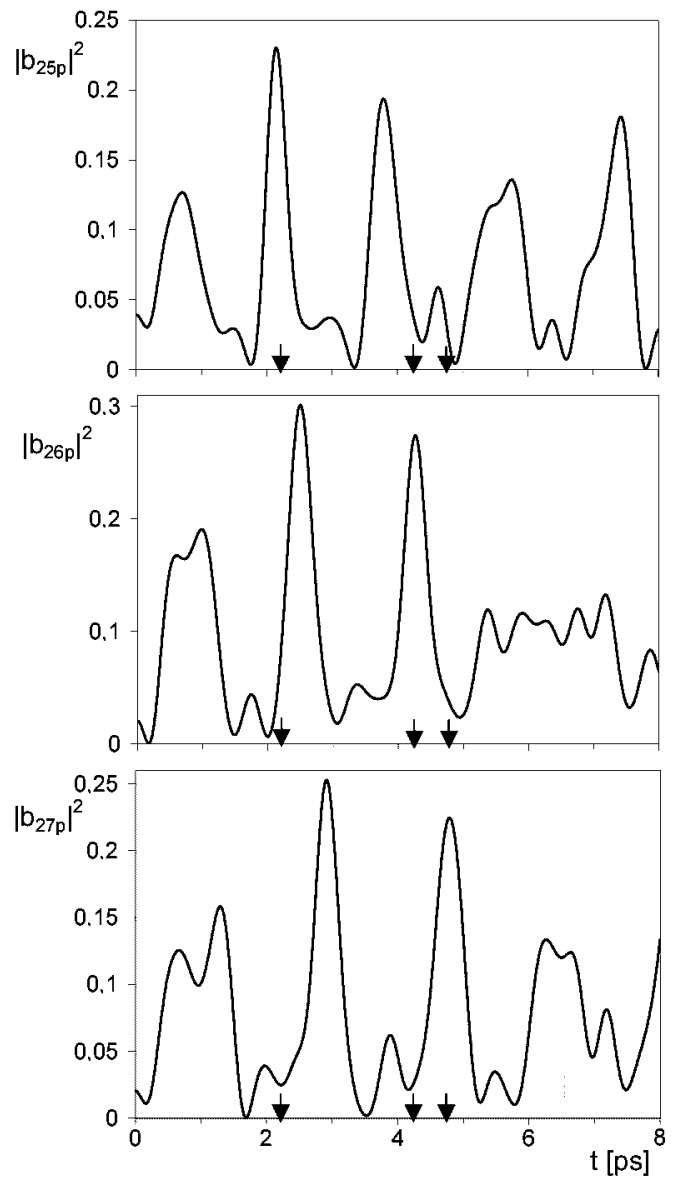

Fig. 2. The after-terahertz-HCP population in different states of the initial caesium $p$-type wave packet versus the initial wave-packet free evolution time $t$, calculated with the use of Eqs. (4) and (2). The arrows point to the specific times (2.1 ps, $4.2 \mathrm{ps}$ and 4.7 ps) for which Fig. 1 is prepared.

between $t_{\mathrm{p}}$ and $t_{\mathrm{K}}$, the amplitude $b_{n^{\prime} 10}=b_{n^{\prime}}$ for the population to be in $\left|n^{\prime} 10\right\rangle$ at the end of the HCP is obtained from Eq. (1) by substituting in place of $|i\rangle$ the wave packet after its free evolution. This results in $b_{n^{\prime}}=\sum_{n=24}^{29} T_{n^{\prime} n} \mathrm{e}^{-\mathrm{i} \omega_{n} t} a_{n}$, where $T_{n^{\prime} n}=T_{n^{\prime} 10, n 10}$ is to be calculated from Eqs. (4) and (2). Figure 1 shows the populations, $\left|b_{n^{\prime}}\right|^{2}$, calculated along this line with the inclusion of quantum defect [6] in both eigenfrequencies $\left(\omega_{n} \rightarrow \omega_{n-\delta}=\omega_{n-3.575}\right)$ and form factors $\left(n \rightarrow n^{*}=n-4\right)$. In Fig. 1 we present the distribution of the population in the initial wave packet, $\left|a_{n}\right|^{2}$, as well as the $p$-state distribution after the HCP, $\left|b_{n^{\prime}}\right|^{2}$, for the experimental free evolution times $t=2.1 \mathrm{ps}, 4.2 \mathrm{ps}$ and $4.7 \mathrm{ps}$. The results of Fig. 1 agree qualitatively with the experimental ones of the original paper by Ahn et al. (Fig. 1 in [7], the first column). They agree nearly quantitatively with 
the results of the calculations by Ahn et al. (also Fig. 1 in [7], the second and third columns) performed along two different lines, one within the short-pulse approximation but with expanding the form factor of Eq. (1) in a series of partial waves (solid lines in our Fig. 1) and the other by direct integration of TDSE (dashed lines) in a basis of 187 states $(n=21-31, l<17, m=0)$. This agreement legitimates the method presented in this paper, based on the analytical parabolic Bersons-Kulsh form factor (Eq. (2)) and its transformation to the spherical basis (Eq. (4)), as an alternative (if not the most convenient) method for theoretical quantum analysis of redistribution of Rydberg state population by HCP's.

In supplement to Fig. 1, we show in Fig. 2 the redistribution by HCP (calculated by the use of Eqs. (4) and (2)) versus time $t$ of free evolution of the initial wave packet (compare Fig. 2 in [7]). The arrows in this figure point to the specific time moments for which we prepared Fig. 1. However, there are also other moments different from those in Fig. 1, for which practically only one state in the initial wave packet survives the HCP. As known from the original analysis by Ahn et al. [7] it is the state in the initial wave packet (marked) that had its phase, $\omega_{n} t$, flipped with respect to all other phases. Such a behaviour of this marked state under the HCP action was originally discussed in [7] in the context of the Grover quantum algorithm, exploiting an inversion about the average, and it was the aim of the present paper to show that the analytical approach based on Eqs. (4) and (2) is able to explain this behaviour in its own way.

The other experiment to which we apply Eqs. (4) and (2) is that by Tielking and Jones [8] on sodium atoms prepared in either the $19 \mathrm{~s}$ or $19 \mathrm{~d}$ states by the use of two tunable ns dye laser pulses. From either of these initial states the population was then redistributed by a nearly Gaussian terahertz HCP of 0.5 ps duration and the redistribution was measured versus electric field in the HCP (versus $Q \approx \varepsilon_{0} t_{\mathrm{p}}$ in the meaning of Eqs. (4) and (2)). In Fig. 3 we present the appropriate redistributions versus $Q$ obtained from Eqs. (4) and (2) with the inclusion of quantum defect $\left(n \rightarrow n^{*}=18\right.$ for $s$ and $p$ states and 19 for $l \geq 2$ states [6]). The highest $\mathrm{Q}$ values in this figure correspond to the highest electric fields included in the original calculations of Tielking and Jones (Figs. 6B and 7B in [8]) based on numerical integration of TDSE in the spherical basis of 200 states. In both cases, i.e., the redistributions from the initial either $19 s$ or $19 d$ states, our curves of Fig. 3 agree at least qualitatively with those of numerical integration of TDSE by Tielking and Jones. The major quantitative differences are: (i) in Fig. 3a, the curve for $19 p$ population is by a factor of 2 higher and the curve for $19 l>2$ is by a factor of 4 lower than the appropriate theoretical curves of Tielking and Jones, (ii) in Fig. 3b, the curve for $19 p$ population does not exhibit oscillations like the theoretical curve of Tielking and Jones (such oscillations do reveal in our calculations when neglecting quantum defect). In the (ii) case, however, our curve with no oscillations seems to be a better imitation of the experimentally measured curve (Fig. 6A in [8]). Besides some mutual quantitative differences both the present curves of Fig. 3 and the the- 

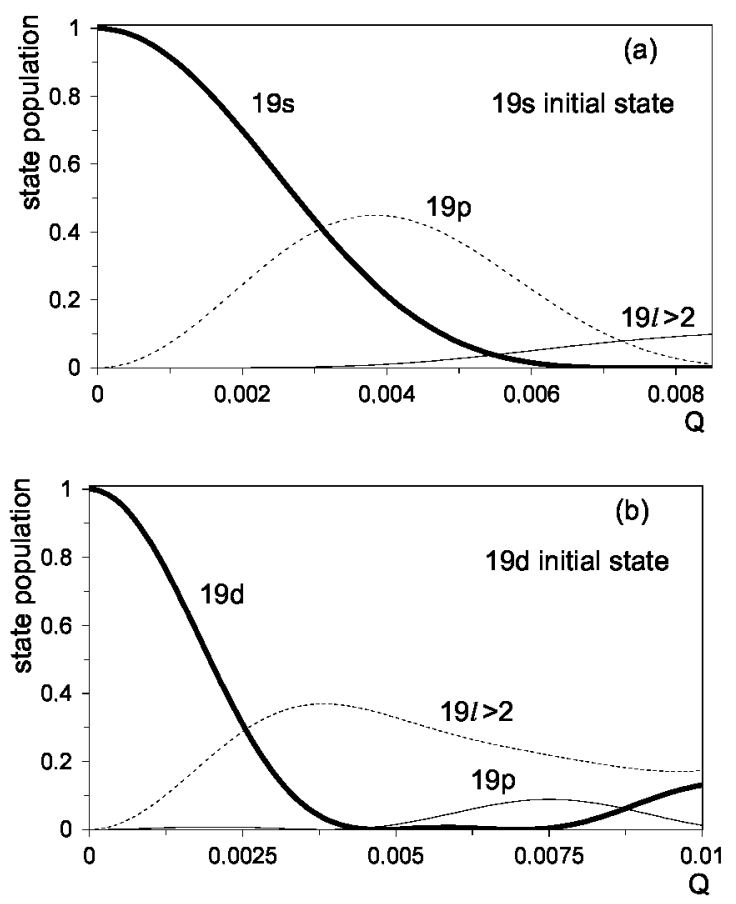

Fig. 3. Population in different sodium states versus momentum $Q$ transferred from the terahertz HCP, calculated from Eqs. (4) and (2), for the $19 \mathrm{~s}$ (a) and $19 d$ (b) initial-state cases, respectively. The notation $l>2$ means total population of all $l>2$ states $(l=$ $3-10$, in practice)

oretical curves of Tielking and Jones reproduce qualitatively the experimentally observed tendencies (Figs. 6A and 7A in [8]). 
The last example to which we apply Eqs. (4) and (2) is a transient redistribution of the hydrogen population from the initial $50 \mathrm{~s}$ state by the optical frequency pulse ( $\omega=0.0735$ a.u.) of peak intensity $10^{9} \mathrm{~W} / \mathrm{cm}^{2}$ and squared sinus envelope of 10 optical periods duration $\left(\sin ^{2}(\tau / 20) \cos \tau, 0 \leq \tau=\omega t \leq 20 \pi\right)$. In this case the rate of changes of the field is $\gamma=\omega$, while the transferred momentum $Q=\left(\varepsilon_{o} / \omega\right) S(\tau)=0.0013\left[\sin \tau-\frac{5}{11} \sin \left(\frac{11}{10} \tau\right)-\frac{5}{9} \sin \left(\frac{9}{10} \tau\right)\right]$. Recently [9], we studied transient redistribution of population by such a pulse by numerical integration of TDSE in a large basis of 1775 spherical states $(n=20-80, l=0-29$, $m=0$ ) and now compare these results with the present results obtained by simply applying analytical Eqs. (4) and (2). Figure 4 shows this comparison for the $\Delta n=0$ transitions, while Fig. 5 for the $\Delta n= \pm 1$ ones. In these figures, full lines are the present short-pulse approximation $\left(t_{\mathrm{p}} / t_{\mathrm{K}} \approx 10^{-4}\right)$ results based on Eqs. (4) and (2), while dots are our previous results of numerical integration of TDSE. There is evident full coincidence between the results of the two methods.

Finally, we point to the most probable source of some quantitative differences, mentioned in the context of Fig. 3, between the present results and those of the numerical integration by Tielking and Jones. The highest experimental fields and those of the Tielking and Jones integration were in fact too high for the short-pulse approximation to perfectly describe the HCP+atom interaction. As known [4], the highest fields of the short-pulse approximation are limited by the requirement for the displacement of the electron by a pulse to be shorter than the electron de Broglie wavelength $\left(\varepsilon_{0}<n \gamma^{2}\right.$ in a.u.). Both in the experiment of Tielking and Jones [8] and Ahn et al. [7] the terahertz HCP fields exceeded by at least a factor of several units those allowed by the short-pulse approximation. Contrary, the optical-frequency pulse of Figs. 4 and 5 has its electric field falling excellently in the applicability domain of the short-pulse approximation, thus the perfect agreement seen therein.

\section{Conclusion}

In this paper we analyzed three examples, being under current research interest, of Rydberg state redistribution by electromagnetic pulses of a duration shorter than the Kepler period of the states involved. In addition to our previous result [3], the results of the present analysis give a much wider basis to conclude that the analytic Bersons-Kulsh form factor (Eq. (2)) of the short-pulse approximation (Eq. (1)) along with its transformation from the original parabolic basis to the spherical one (Eq. (4)) is an effective and convenient method of quantum calculations of the redistribution by both terahertz half-cycle and optical frequency many-cycle pulses, able to compete with the hitherto preferred method of numerical solution of the time-dependent Schrödinger equation in a selected-state basis.

\section{Acknowledgment}



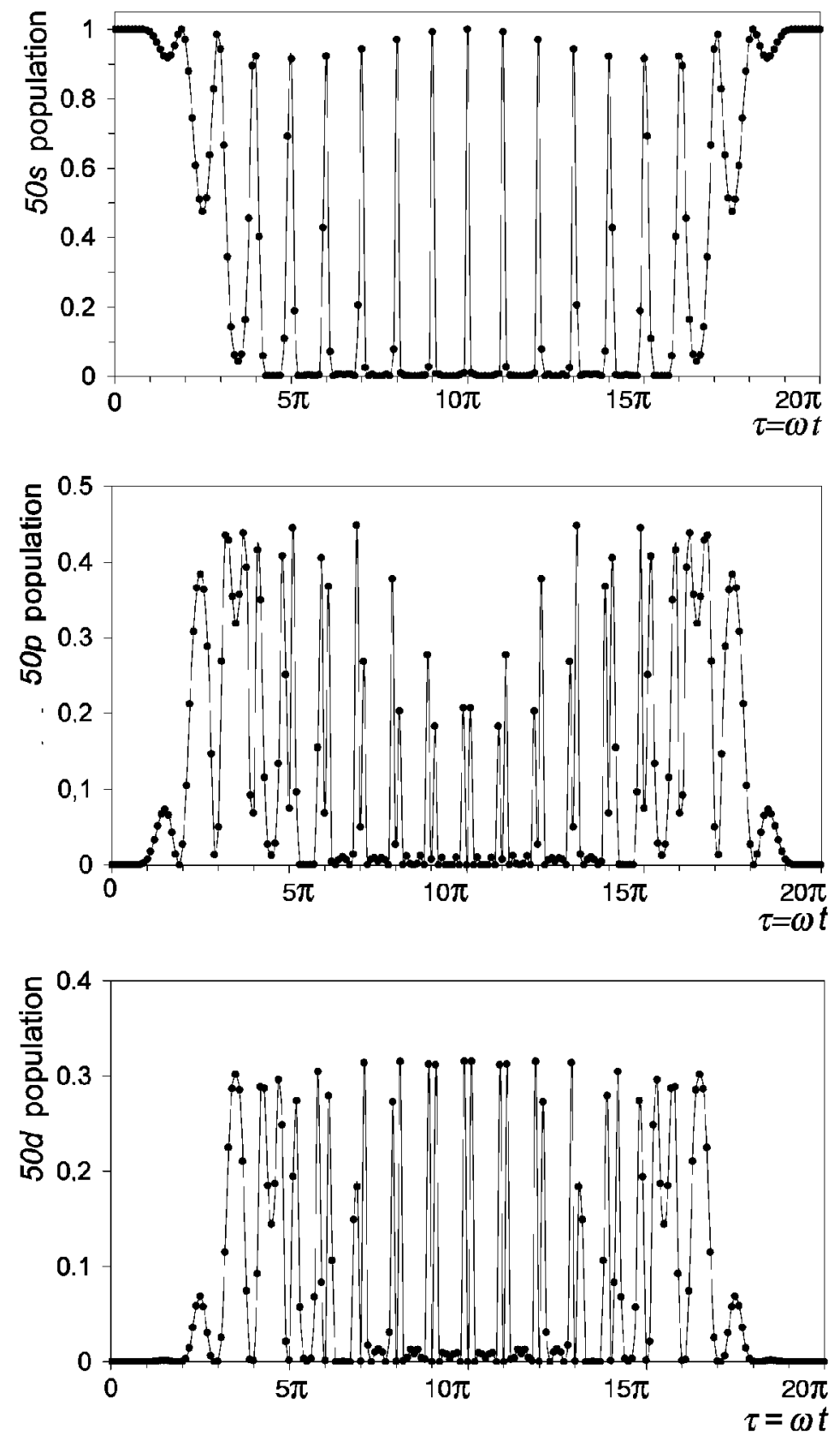

Fig. 4. Hydrogen state population versus evolution time of the optical frequency pulse $(\omega=0.0735$ a.u. $)$ of the $\sin ^{2}(\tau / 20) \cos \tau$ shape, 10 cycle duration $(0 \leq \tau=\omega t \leq 20 \pi)$ and $10^{9} \mathrm{~W} / \mathrm{cm}^{2}$ peak intensity. The case of the $\Delta n=0$ transitions from the initial $50 \mathrm{~s}$ state. Full lines - the present analytic results, dots - the results of our numerical integration of TDSE. 

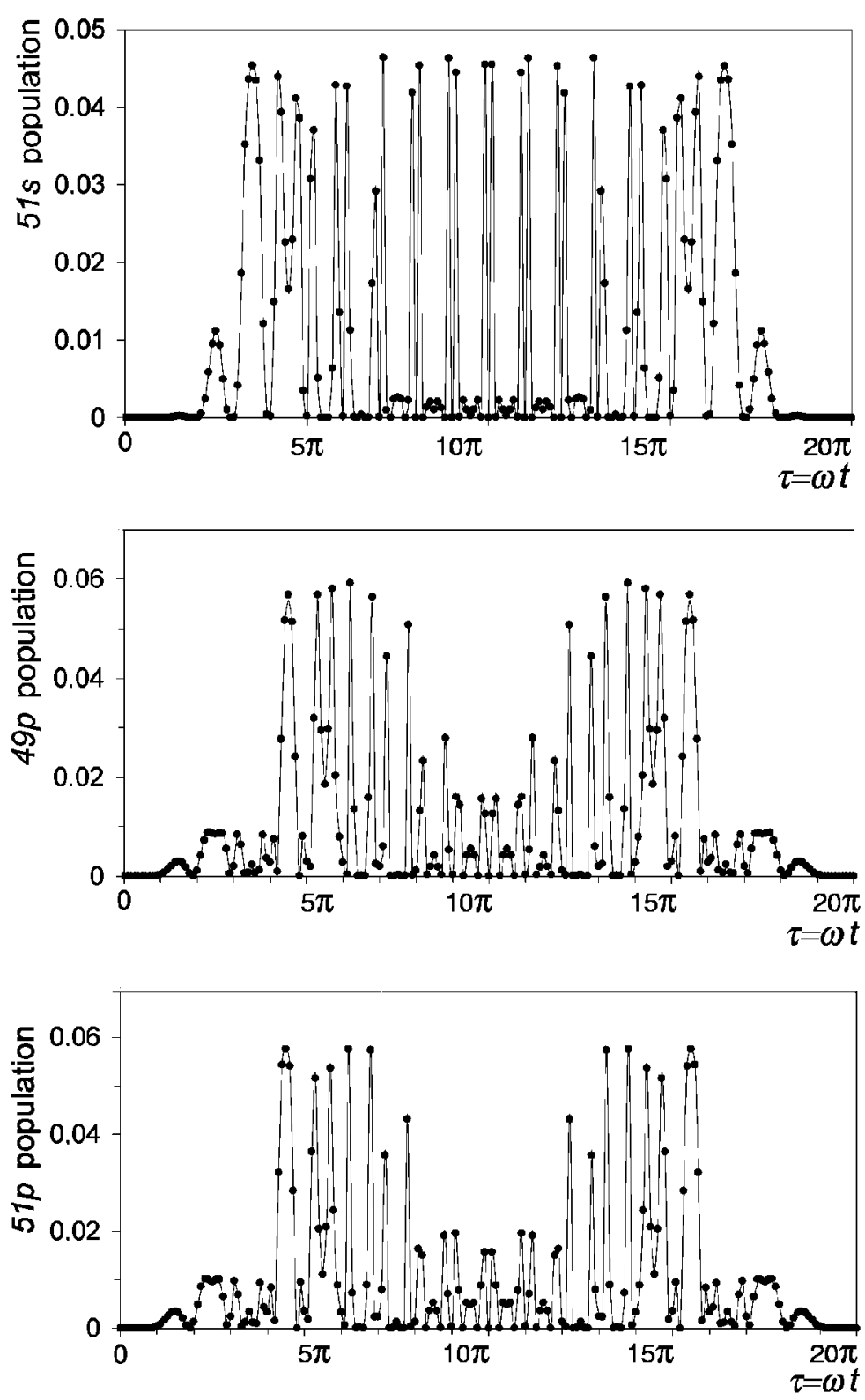

Fig. 5. The same as in Fig. 4 but for the $\Delta n= \pm 1$ transitions.

One of us (M.S.) thanks the State Committee for Scientific Research for support under grant No. 2 PO3B 00522.

References 
[1] P.H. Bucksbaum, The Physics and Chemistry of Wave Packets, Eds. J.A. Yeazell, T. Uzer, Wiley, New York 2000, p. 159; C. Wesdorp, F. Robicheaux, L.D. Noordam, Phys. Rev. Lett. 87, 083001 (2001).

[2] A. Wetzels, A. Gürtler, H.G. Muller, L.D. Noordam, Eur. Phys. J. D 14, 157 (2001).

[3] R. Parzyński, M. Sobczak, Phys. Rev. A 65, 045401 (2002).

[4] P. Krstic, Y. Hahn, Phys. Rev. A 48, 4515 (1993); Phys. Rev. A 50, 4629 (1994); Phys. Lett. A 192, 47 (1994).

[5] I. Bersons, A. Kulsh, Phys. Rev. A 55, 1674 (1997).

[6] T.F. Gallagher, Rydberg Atoms, Cambridge University Press, Cambridge 1994, p. 76 (Eq. 6.18), 353.

[7] J. Ahn, D.N. Hutchinson, C. Rangan, P.H. Bucksbaum, Phys. Rev. Lett. 86, 1179 (2001).

[8] N.E. Tielking, R.R. Jones, Phys. Rev. A 52, 1371 (1995).

[9] R. Parzyński, M. Sobczak, A. Wójcik, Phys. Rev. A 63, 063413 (2001).

[10] M. Inokuti, Rev. Mod. Phys. 43, 297 (1971).

[11] A.O. Barut, R. Wilson, Phys. Rev. A 40, 1340 (1989). 